\title{
The Ron/STK receptor tyrosine kinase is essential for peri-implantation development in the mouse
}

\author{
Rebecca S. Muraoka, ${ }^{1}$ William Y. Sun, ${ }^{2}$ Melissa C. Colbert,${ }^{3}$ Susan E. Waltz, ${ }^{2}$ \\ David P. Witte, ${ }^{4}$ Jay L. Degen, ${ }^{2}$ and Sandra J. Friezner Degen ${ }^{2}$ \\ ${ }^{1}$ Graduate Program in Developmental Biology, University of Cincinnati College of Medicine, Cincinnati, Ohio 45229-3039, USA \\ ${ }^{2}$ Division of Developmental Biology, \\ ${ }^{3}$ Division of Molecular Cardiovascular Biology, and \\ ${ }^{4}$ Division of Pathology, Children's Hospital Research Foundation, Cincinnati, Ohio 45229-3039, USA \\ Address correspondence to: Sandra J.F. Degen, Division of Developmental Biology, Children's Hospital Research Foundation, \\ 3333 Burnet Avenue, Cincinnati, Ohio 45229-3039, USA. Phone: (513) 636-4816; Fax: (513) 636-4317; \\ E-mail: sandra.degen@chmcc.org
}

Rebecca S. Muraoka's present address is: Department of Immunology and Microbiology, Vanderbilt University School of Medicine, Nashville, Tennessee 37235, USA.

Received for publication December 17, 1998, and accepted in revised form March 26, 1999.

\begin{abstract}
The Ron/STK receptor tyrosine kinase is a member of the c-Met family of receptors and is activated by hepatocyte growth factor-like protein (HGFL). Ron activation results in a variety of cellular responses in vitro, such as activation of macrophages, proliferation, migration, and invasion, suggesting a broad biologic role in vivo. Nevertheless, HGFL-deficient mice grow to adulthood with few appreciable phenotypic abnormalities. We report here that in striking contrast to the loss of its only known ligand, complete loss of Ron leads to early embryonic death. Embryos that are devoid of Ron ( $\left.\mathrm{Ron}^{-/}\right)$are viable through the blastocyst stage of development but fail to survive past the peri-implantation period. In situ hybridization analysis demonstrates that Ron is expressed in the trophectoderm at embryonic day (E) 3.5 and is maintained in extraembryonic tissue through E7.5, compatible with an essential function at this stage of development. Hemizygous mice $\left(\mathrm{Ron}^{+/}\right)$grow to adulthood; however, these mice are highly susceptible to endotoxic shock and appear to be compromised in their ability to downregulate nitric oxide production. These results demonstrate a novel role for Ron in early mouse development and suggest that Ron plays a limiting role in the inflammatory response.
\end{abstract}

J. Clin. Invest. 103:1277-1285 (1999).

\section{Introduction}

Receptor tyrosine kinases (RTKs) are responsible for transducing signals from the cell surface to the intracellular environment. One distinct family of RTKs whose biologic significance remains to be fully defined is composed of the proto-oncogene products c-Met and Ron. These heterodimeric cell surface receptors are distinguished by a ligand-binding extracellular domain and a highly conserved intracellular domain that harbors the catalytic tyrosine kinase activity (1-4). Although the cMet receptor is well characterized with regard to its ability to stimulate motility, proliferation, and morphologic changes in many epithelial cell types, and is known to be essential for liver development and embryonic survival, little is known regarding the physiologic responses initiated by Ron (5-7).

The ligand that binds to Ron is hepatocyte growth factor-like protein/macrophage stimulating protein (HGFL/MSP; refs. 8-11). This growth factor family also includes hepatocyte growth factor (HGF), the ligand that activates c-Met. Binding of Ron by HGFL results in increased tyrosine kinase activity, allowing for signal transduction to the intracellular environment through a multifunctional, bidentate binding site. The tyrosine kinase activity of Ron is normally inactive in the absence of HGFL, but overexpression of Ron or the expression of a naturally occurring truncated splice variant of Ron results in constitutive tyrosine kinase activity and is associated with an increase in the invasive capacity of various tumor cell lines (12-13). Based on this observation, it is thought that Ron may play a fundamental role in cellular invasion and motility.

Ron is thought to be involved in mediating a variety of physiologic processes, particularly in regard to the development or function of certain hematopoietic cells (4, 14-16). The mouse homologue of Ron is synthesized in various hematopoietic-derived cells of the adult mouse, including peritoneal macrophages, osteoclasts, and megakaryocytes (17-19). HGFL induces the activation of peritoneal macrophages, stimulating phagocytosis, morphologic changes, and chemotactic migration (20). Ron inhibits expression of inducible nitric oxide synthase (iNOS) in peritoneal macrophages in vitro, thus inhibiting the synthesis of nitric oxide (NO) produced in response to bacterial endotoxins (21). Therefore, it is proposed that Ron may regulate the inflammatory response of macrophages. In vitro evidence suggests that Ron increases DNA synthesis, ruffled border formation, and bone resorption by osteoclasts, and enhances megakaryocyte maturation. Ron expression has also been detected in the gastrointestinal tract, brain, and skin of the adult mouse, and Ron stimulates the prolif- 
eration and motility of keratinocytes in vitro. The functions of Ron in vivo are not yet understood, but these findings suggest its involvement in a wide spectrum of biologic activities.

An equally complex pattern of Ron expression is observed in the developing mouse, implying a functional requirement for Ron during embryogenesis. Ron expression is detected at embryonic day (E) 12.5 in the liver, perhaps reflecting fetal hematopoiesis, and in the central nervous system, primarily in the spinal and dorsal root ganglia. By E14.5, Ron expression has been initiated in the digestive tract epithelium, skin keratinocytes, and bone $(22,23)$. Information is not available on the expression of Ron prior to E7.5 in the mouse or on its expression in extraembryonic tissue.

In spite of the growing body of evidence that Ron and HGFL are involved in embryogenesis and/or inflammation, HGFL-deficient mice grow to adulthood with few ascertainable defects, aside from the accumulation of lipid-filled cytoplasmic vesicles in hepatocytes, which does not perturb synthetic or secretory hepatic function (24). Activation of macrophages is delayed, but nevertheless attained, in the absence of HGFL, and hematopoiesis and wound healing appear to be unal- tered in mice that are devoid of HGFL. To directly determine the specific functions of Ron in development and in the adult mouse, we have examined the consequences of Ron gene disruption in mice. In contrast to the loss of the ligand HGFL, we report that the complete loss of the Ron receptor tyrosine kinase leads to death in the periimplantation stage of development.

\section{Methods}

Disruption of the mouse Ron gene. In construction of the targeting vector, exons 1-14 and part of exon 15 of the mouse Ron gene, isolated from a 129/Ola genomic library (15), were replaced by a 6.0-kb hypoxanthine phosphoribosyltransferase (HPRT) expression cassette, which also serves as a positive selection marker (Figure 1a). A 2.0-kb XbaI-Bam HI fragment from the 5' flanking sequence was used as the short arm of the targeting vector and was cloned into the XbaI and BamHI sites of a Bluescript plasmid (Stratagene, La Jolla, California, USA). A 3.1-kb SmaI fragment from the $3^{\prime}$ end of the mouse Ron gene, including part of exon 15 and exons 16-18, was used as the long arm of the targeting vector and was cloned into the SmaI site of the Bluescript plasmid containing the short arm. The HPRT expression cassette was cloned into the Bam HI site of the Bluescript plasmid. A $2.0-\mathrm{kb}$ cassette coding for the herpes simplex virus thymidine kinase gene (HSV-tk) was cloned into the
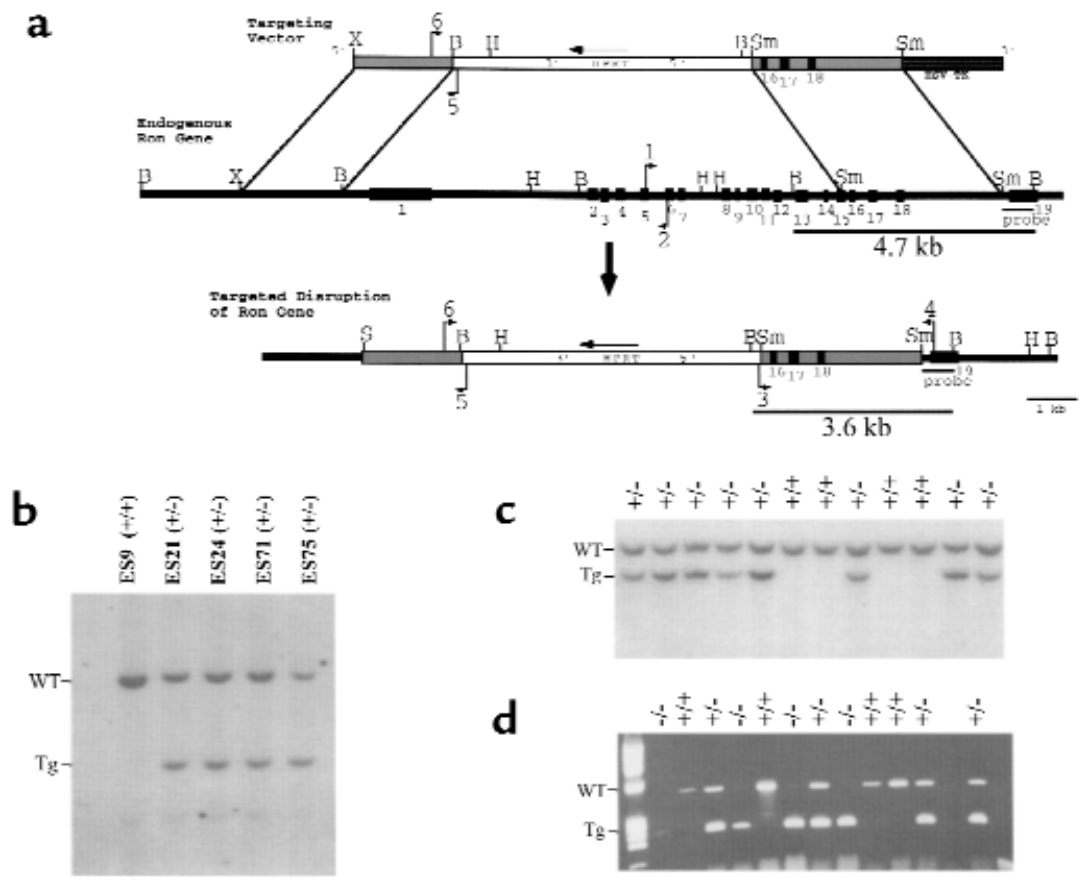

\section{Figure 1}

Disruption of the mouse Ron gene in embryonic stem (ES) cells and mice. (a) Schematic representation and partial restriction map of the targeting vector (top) used to disrupt the endogenous mouse Ron gene (middle). The predicted organization of the targeted mouse Ron gene following homologous recombination is also shown (bottom). The targeting vector contains sequences homologous to the endogenous Ron gene (gray bars) flanking the HPRT expression cassette (white bar). The HSV-tk gene flanks the targeting vector at the $3^{\prime}$ end (dark gray bar). The exons of the endogenous Ron gene are numbered (1-19, black boxes). The primers used for PCR genotyping are indicated with arrows and are numbered 1-6. The probe used for Southern blot analysis is shown below the endogenous Ron gene. The BamHI fragments of genomic DNA that are complementary to the probe are indicated with the expected sizes (4.7 kb and $3.6 \mathrm{~kb}$ ). B, BamHI. H, HindIII. S, Sall Sm, Smal. X, Xbal. (b) Southern blot analysis of BamHI-digested DNA from selectionresistant ES cell clones. The wild-type allele (WT) is represented by a 4.7-kb fragment, while the targeted allele (Tg) is represented by a 3.6-kb fragment. ES9 was included as a wild-type control. (c) Southern blot analysis of BamHI-digested DNA from a single litter generated from a Ron $/$ - intercrossing. Offspring were genotyped at 7 days postnatal. Of the 12 littermates shown, 8 are $\operatorname{Ron}^{+/-}(+/-)$and 4 are Ron ${ }^{+/+}(+/+)$. (d) Genotype analysis by PCR of a single litter of E3.5 blastocysts generated from a Ron ${ }^{+/-}$intercrossing. The wild-type allele is represented by a 523 -bp product generated from primers 1 and 2 (see a); the targeted allele is represented by a 274-bp product generated from primers 5 and 6. 
HindIII and SalI sites of the Bluescript vector and used as a negative selection marker. The targeting vector was linearized with NotI prior to electroporation. The linearized targeting vector was introduced into mouse embryonic stem (ES) cells as previously described (24).

Genotype analysis. Southern blot analysis of BamHI-digested genomic DNA from selection-resistant ES cell clones or mouse tail biopsies was performed as described previously (24). The probe used for Southern blot analysis is derived from a region of the Ron gene that lies outside the proposed region of homologous recombination. Alternatively, genotype analysis on DNA from tail or ear biopsy was performed using a multiplex PCR reaction (24). The endogenous Ron allele was detected using primers 1 (5'-CCCTGCCGACTGCTGCCTAAGGACT-3', exon $5)$ and 2 (5'-AGAGATGGCTTCCACTCGGAAGTGC-3', exon 6), generating a 523-bp product (Figure 1a). The targeted Ron allele was detected by primers 3 (5'-CGTCAGGCCCGAGCCCGCCGGCCCGGGTCCC-3', inside the HPRT expression cassette) and 4 (5'-CTGTACCTGCTCCGGAGGCACACTC-3', exon 19, outside the region of homologous recombination), resulting in a 3.56-kb PCR product (Figure 1a). Once the targeted allele had been established in the $\mathrm{F}_{1}$ offspring by Southern blot analysis, primers 5 (5'-TATTACCAGTGAATGTTTGTCAGCAGTTCCC$3^{\prime}$, inside the HPRT expression cassette) and 6 (5'TGTACCAGGGCACCGGAGAGACCGA-3', Ron 5' flanking sequences) were used, generating a 274-bp product (Figure 1a).

Generation of mouse colonies. Targeted ES cells were injected into C57Bl/6 blastocysts and transferred into pseudopregnant $\mathrm{C} 57 \mathrm{Bl} / 6$ females to generate chimeric founders (performed by the ES Cell Gene Altered Mouse Service at the University of Cincinnati College of Medicine). Chimeric founders were crossed to NIH Black Swiss mice to generate $\mathrm{F}_{1}$ offspring. Hemizygous $\left(\right.$ Ron $\left.^{+/-}\right) \mathrm{F}_{1}$ males were crossed to NIH Black Swiss females to generate $\mathrm{F}_{2}$ offspring. Ron ${ }^{+/-} \mathrm{F}_{2}$ siblings were intercrossed to generate $\mathrm{F}_{3}$ offspring. All experiments described were performed in $\mathrm{F}_{3}$ offspring of the 129/Black Swiss mixed genetic background mice, except where indicated. For the 129/CD-1 mixed genetic background experiment, chimeric founders were crossed with CD-1 females to generate $F_{1}$ offspring. CD-1/129 $F_{1}$ offspring were intercrossed to produce the experimental $\mathrm{F}_{2}$ generation. The targeted Ron allele was also introduced into a $\mathrm{C} 57 \mathrm{Bl} / 6$ background by crossing a chimeric founder with a C57Bl/ 6 mouse, followed by backcrossing to $\mathrm{C} 57 \mathrm{Bl} / 6$ mice for 6 generations.

Developmental analysis. Offspring from timed matings between $\mathrm{Ron}^{+/-}$mice were isolated at specific developmental time points (Table 1). Prior to parturition, embryos were dissected cleanly away from potentially contaminating maternal tissue. Embryos isolated at E5.5 through E12.5 were genotyped by Southern blot analysis of whole-embryo DNA, or alternatively, genotyped by PCR analysis of yolk sac or whole-embryo DNA. For PCR analysis, the yolk sacs or embryos were digested in $10-100 \mu \mathrm{l}$ of digestion buffer $(500 \mathrm{mM} \mathrm{KCl}, 100 \mathrm{mM}$ Tris- $\mathrm{HCl}$ [pH 8.3 ], $0.1 \mathrm{mg} / \mathrm{mL}$ gelatin, $0.45 \%$ Nonidet P-40, $0.45 \%$ Tween-20, and $0.1 \mathrm{mg} / \mathrm{mL}$ proteinase $\mathrm{K}$ ) for 16 hours at $55^{\circ} \mathrm{C}$, and the lysate was used for PCR analysis. Blastocysts isolated at E3.5 were flushed from uterine horns and individually cultured for 1 week in DMEM supplemented with 15\% FBS and $4 \mathrm{mM}$ L-glutamine.

Histologic analyses. Pregnant mice from timed matings between Ron ${ }^{+/-}$mice at E6.5 and E7.5 were sacrificed, and the entire gravid uterus was harvested, fixed in $10 \%$ buffered formalin, and cut into segments prior to embedding in paraffin. The entire embedded uterine segments were serially sectioned, mounted on slides, and stained with hematoxylin and eosin. All slides were examined microscopically.

In situ bybridization. Decidua containing the undisturbed mouse embryos were isolated at E7.5 and fixed in 4\% paraformaldehyde in PBS ( $\mathrm{pH}$ 7.4). Blastocysts were flushed
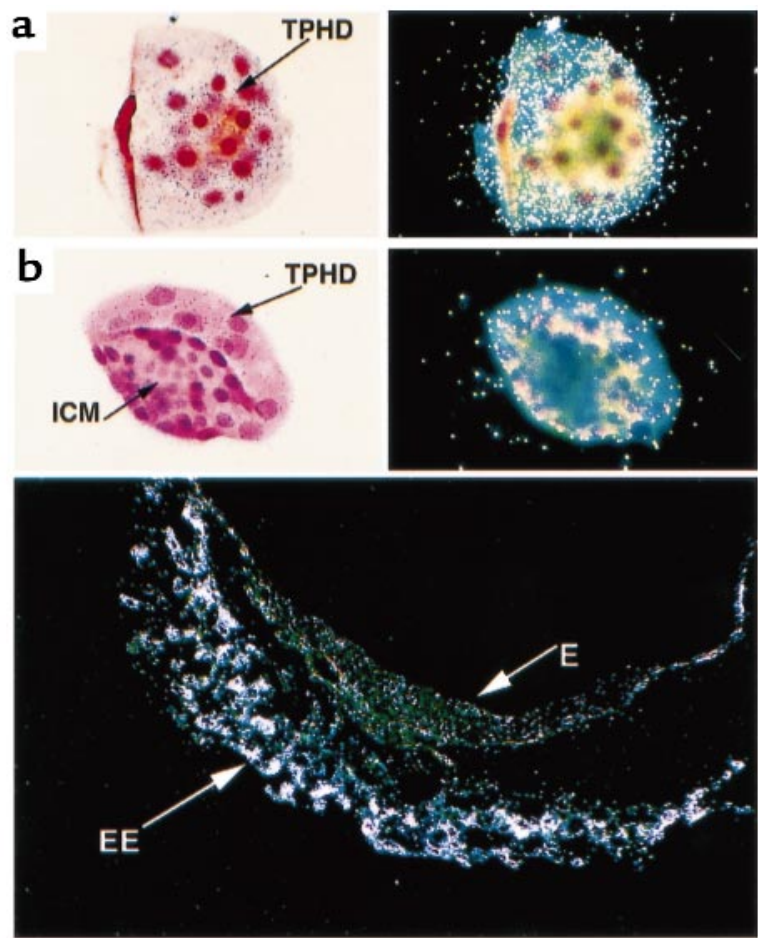

\section{Figure 2}

In situ hybridization of wild-type embryos at E3.5 and E7.5. The embryos were stained with hematoxylin and eosin and hybridized with a probe complementary to the $3^{\prime}$ end of the mouse Ron transcript. Two distinct orientations of the blastocysts are shown: (a) orientation highlighting the trophectoderm of the blastocysts, and (b) orientation highlighting the inner cell mass. The light-field exposure is shown in the left panels, and the dark-field exposure is shown in the right panels. The bottom panel shows the dark-field exposure of a wild-type embryo isolated with the intact decidua at E7.5. TPHD, trophectoderm. ICM, inner cell mass. EE, extraembryonic tissue. E, embryonic tissue.

from uterine horns, fixed directly into $4 \%$ paraformaldehyde, and centrifuged directly onto slides. A ${ }^{35}$ S-labeled, 1,100nucleotide Ron antisense RNA probe from the $3^{\prime}$ end of mouse Ron cDNA and a 900-nucleotide Ron sense RNA control were synthesized and individually hybridized to cryosections of fixed tissue as described previously $(15,25)$.

Northern and Western blot analysis. Freshly harvested mouse tissues were homogenized in $200 \mathrm{mM}$ Tris- $\mathrm{HCl}$ ( $\mathrm{pH} 8.0), 0.2 \%$ SDS, and $0.5 \%$ Triton X-100, and centrifuged at 15,000 rpm for 30 minutes at $4^{\circ} \mathrm{C}$. Protein $(20 \mu \mathrm{g})$ from the supernatant was analyzed for the presence of HGFL or Ron by Western blot analysis as described (26). HGFL was detected using a previously characterized rabbit polyclonal antibody against its single-chain form (10), and Ron was detected using a goat polyclonal antibody against the extracellular domain of mouse Ron (R\&D Systems Inc., Minneapolis, Minnesota, USA). Specific antibody binding was visualized using a chemiluminescent system (ECL; Amersham Life Sciences Inc., Arlington Heights, Illinois, USA). For Northern blot analysis, total RNA was isolated from freshly harvested mouse liver, size fractionated, and transferred to a nylon membrane (GeneScreen Plus; Du Pont NEN Research Products, Boston, Massachusetts, USA). A full-length mouse HGFL cDNA (8) was labeled with $\left[\alpha_{-}{ }^{32} \mathrm{P}\right] \mathrm{dCTP}$ and used as a probe, as described previously (24).

Production of HGFL. The production of recombinant HGFL was carried out by transfection of Chinese hamster ovary (CHO) cells with wild-type human HGFL cDNA cloned into pcDNA3, as described (26). This recombinant human HGFL 
has been previously shown to cause the activation of mouse peritoneal macrophages in a manner that is identical to the action of mouse HGFL (26).

Isolation, culture, activation, and measurement of $\mathrm{NO}$ of mouse peritoneal macrophages. Adult mouse peritoneal cavities were lavaged with $10 \mathrm{~mL}$ sterile, serum-free DMEM. Cells were centrifuged and plated in $35-\mathrm{mm}$ wells with $10^{6}$ cells per well. Peritoneal macrophages were treated with culture media from wild-type $\mathrm{CHO}$ cells or $\mathrm{CHO}$ cells expressing human HGFL. Cell morphology was observed at timed intervals thereafter using a phase-contrast microscope. Cells displaying a compact, spindleshaped morphology with elongated cytoplasmic processes were considered activated. The percentage of activated macrophages per field was determined by blinded counting. The synthesis of NO by peritoneal macrophages was determined by measuring levels of nitrite, a stable by-product of NO, in the serum-free culture media of plated macrophages. The cells were treated with 1 $\mu \mathrm{g} / \mathrm{mL}$ LPS from Escherichia coli (Sigma Chemical Co., St. Louis, Missouri, USA) and/or $100 \mathrm{U} / \mathrm{mL}$ recombinant mouse IFN- $\gamma$ (Sigma Chemical Co.) in the presence or absence of 50-100 $\mathrm{ng} / \mathrm{mL}$ human HGFL (see above). Culture media $(100 \mu \mathrm{L})$ from the stimulated macrophages were added to $100 \mu \mathrm{l}$ Greiss reagent (1\% sulfanilamide and $0.1 \%$ naphthylethylene diaminehydrochloride in $2.5 \% \mathrm{H}_{3} \mathrm{PO}_{4}$; Promega Corp., Madison, Wisconsin, USA). The absorbance of the resulting colorimetric reaction was measured at $540 \mathrm{~nm}$. The nitrite levels in culture media were calculated by comparison with a standard curve. All samples were assayed in triplicate.

Challenge with LPS. Wild-type or Ron ${ }^{+/-}$mice were administered LPS $(25 \mu \mathrm{g} / \mathrm{g}$; Sigma Chemical Co. $)$ in sterile PBS $(1 \mathrm{mg} / \mathrm{mL})$ by peritoneal injection (27). Mouse viability was determined at 24hour intervals for 14 days following the administration of LPS.

\section{Results}

Disruption of the mouse Ron gene in ES cells and mice. Disruption of the mouse Ron gene was performed in ES cells by homologous recombination (Figure 1a). Targeting resulted in the deletion of 300 bases upstream of exon 1 through the middle of exon 15 , a region that encompasses the entire extracellular domain and transmembrane domain of the Ron gene product. Southern blot analysis

Table 1

Genotype distribution of offspring from Ron $^{+/-}$intercrossings

\begin{tabular}{|c|c|c|c|c|c|}
\hline \multirow[t]{2}{*}{ Age } & \multirow[t]{2}{*}{ Total } & \multicolumn{4}{|c|}{ Genotype } \\
\hline & & $+/+$ & $+/-$ & $-1-$ & $\chi^{2}$ \\
\hline \multicolumn{6}{|c|}{ (NIH Black Swiss/129) } \\
\hline E3.5 & 48 & $12(25 \%)$ & $25(52 \%)$ & $11(23 \%)$ & $P>0.9$ \\
\hline E5.5 & 35 & $7(20 \%)$ & $21(60 \%)$ & $7(20 \%)$ & $P>0.4$ \\
\hline E6.5 & 50 & $15(30 \%)$ & 33 (66\%) & $2(4 \%)$ & $P<0.003$ \\
\hline E7.5 & 62 & $22(35.5 \%)$ & 40 (64.5\%) & 0 & $P<0.001$ \\
\hline E8.5 & 65 & $23(35.4 \%)$ & $42(64.6 \%)$ & 0 & $P<0.001$ \\
\hline E10.5 & 71 & $23(32.4 \%)$ & $48(67.6 \%)$ & 0 & $P<0.001$ \\
\hline E12.5 & 44 & $15(34.1 \%)$ & $29(65.9 \%)$ & 0 & $P<0.001$ \\
\hline Term pups & 390 & $129(33.1 \%)$ & $261(66.9 \%)$ & 0 & $P<0.001$ \\
\hline \multicolumn{6}{|l|}{$(C D-1 / 129)$} \\
\hline Term pups & 116 & $40(34.5 \%)$ & $76(65.5 \%)$ & 0 & $P<0.001$ \\
\hline \multicolumn{6}{|l|}{$(\mathrm{C} 57 \mathrm{Bl} / 6)$} \\
\hline Term pups & 87 & $28(32.2 \%)$ & $59(67.8 \%)$ & 0 & $P<0.001$ \\
\hline
\end{tabular}

The genotype was determined by Southern blot analysis or PCR, as described in Methods. The genotype percentage for each developmental time point is given in parentheses. $P$ values were determined using $\chi^{2}$ analysis. identified 9 ES cell clones that contained the targeted Ron allele out of 114 selection-resistant clones screened (Figure 1b). Three independently derived ES clones were used to generate chimeric founders that were found to transmit the targeted Ron allele to their offspring.

Homozygous loss of Ron is embryonic lethal prior to E6.5. To examine the consequences of the targeted loss of both Ron alleles, hemizygous siblings $\left(\mathrm{Ron}^{+/-}\right)$were intercrossed and the genotypes of the progeny were determined by Southern blot analysis (Figure 1c). The transmission of the mutant Ron allele did not follow a Mendelian pattern of inheritance (Table 1). In total, $33.1 \%$ of the pups were wild-type $\left(\mathrm{Ron}^{+/+}\right)$, while $66.9 \%$ of the pups were $\mathrm{Ron}^{+/-}$. No homozygous mutant $\left(\right.$ Ron $\left.^{-/-}\right)$mice were identified, indicating that the expected proportion of Ron $^{-/}$littermates dies at some point during embryonic development. This intrauterine death was observed for mice derived from all 3 ES cell lines used to generate gene-targeted mice.

To precisely identify the time frame in which $\mathrm{Ron}^{-/-}$ embryos undergo developmental failure, litters from $\mathrm{Ron}^{+/-}$intercrosses were dissected cleanly from the uterus, microscopically examined, and genotyped at specific time points during gestation (Table 1). As early as E7.5, the ratio of $\mathrm{Ron}^{+/+}$to $\mathrm{Ron}^{+/-}$embryos was approximately 1:2 (Table 1), but Ron ${ }^{-/}$embryos were not identified at this or later time points, suggesting that $\mathrm{Ron}^{-/}$ embryos die prior to E7.5. The Ron ${ }^{+/-}$embryos were phenotypically indistinguishable from their wild-type littermates upon gross morphologic examination.

To determine if $\mathrm{Ron}^{-/-}$embryos develop to at least the blastocyst stage, at E3.5 blastocysts were flushed from the uterine horns of $\mathrm{Ron}^{+/-}$females bred to $\mathrm{Ron}^{+/-}$ males, and individually cultured. The cultured blastocysts were monitored daily over a period of 7 days and then genotyped by PCR analysis (Figure 1d). At this stage of development, all 3 possible genotypes were observed, and the expected Mendelian ratio of inheritance was recovered (Table 1). Ron $^{-/-}$embryos were not morphologically distinguishable from their $\mathrm{Ron}^{+/+}$or $\mathrm{Ron}^{+/-}$counterparts. Ron ${ }^{-/}$ blastocysts were able to hatch from the zona pelluci$\mathrm{da}$ in culture, and the trophoblast outgrowths were able to attach to the tissue culture plate. The inner cell masses of $\mathrm{Ron}^{-/-}$blastocysts appeared normal and proliferated at approximately the same rate as the inner cell masses derived from $\mathrm{Ron}^{+/+}$ blastocysts, as determined by daily visual observation of blastocyst growth.

Analysis of embryos collected from $\mathrm{Ron}^{+/-}$breeding pairs later in gestation indicated that $\mathrm{Ron}^{-/}$embryos continued to develop at least until E5.5. The frequency of $\mathrm{Ron}^{-/}$ embryos at E5.5 was found to be $20 \%$, close to what is expect- 

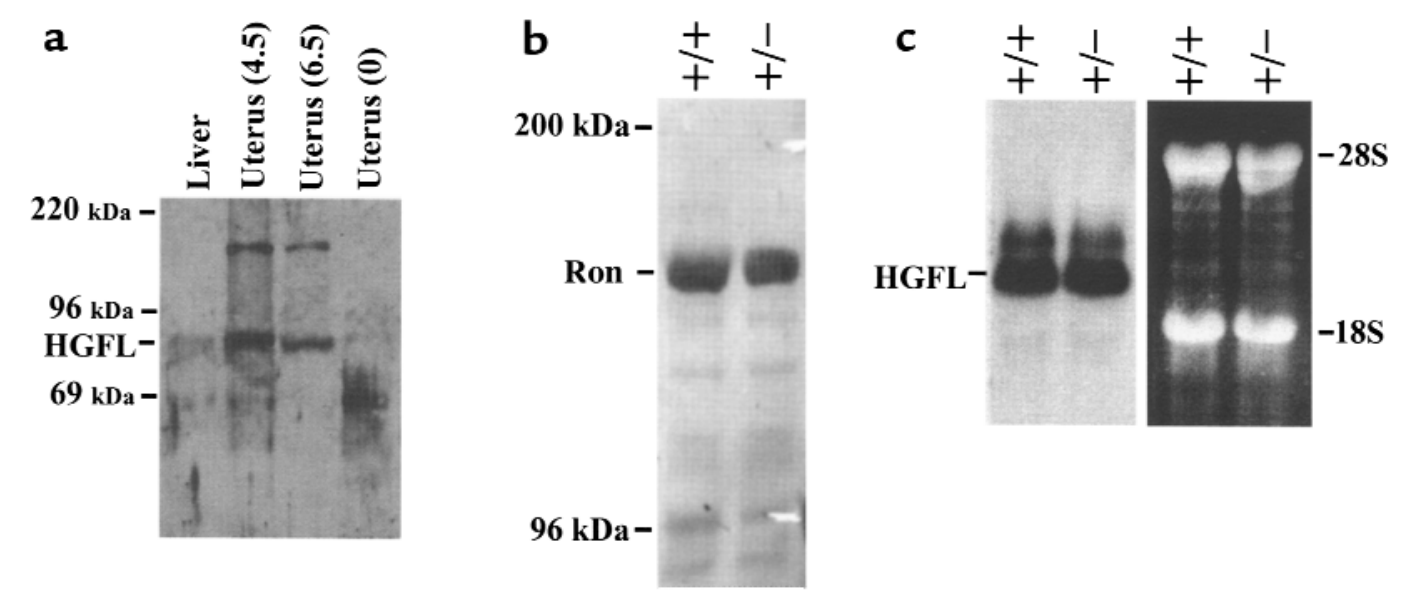

\section{Figure 3}

Expression of HGFL in the liver and maternal uterus. (a) Expression of HGFL in the pregnant uterus. Uterine tissue was dissected from pregnant wildtype females at 4.5 and 6.5 days postcoitus, or from virgin (0) females. Liver was dissected from virgin females as a positive control for HGFL expression. Proteins were electrophoresed and Western blot analysis was performed. The expected size of HGFL ( $80 \mathrm{kDa})$ is indicated, as are the sizes of the molecular weight markers. The identity of the higher-molecular-weight band is unknown, but this band does not correspond to the size expected for HGFL. (b) Expression of Ron in the liver of $\mathrm{Ron}^{+/}$and $\mathrm{Ron}^{+/-}$mice. Western blot analysis was performed on protein isolated from the livers of wild-type and $\mathrm{Ron}^{+/-}$mice. The expected size of Ron $(\sim 170 \mathrm{kDa})$ is indicated, as are the sizes of the molecular weight markers. (c) Expression of HGFL RNA in the livers of $\mathrm{Ron}^{+/+}$and $\mathrm{Ron}^{+/-}$mice. Total RNA was isolated from the livers of $\mathrm{Ron}^{+/+}$and $\mathrm{Ron}^{+/-}$mice and analyzed for expression of HGFL mRNA by Northern blot analysis. The position of the mouse HGFL mRNA is indicated, as are the positions of the $18 \mathrm{~S}$ and $28 \mathrm{~S}$ ribosomal RNAs. The ethidium bromide-stained agarose gel is shown (at right) to indicate the equivalent loading of samples.

ed based on a normal Mendelian transmission pattern. In contrast, at E6.5 only $4.0 \%$ of the embryos analyzed were found to be $\mathrm{Ron}^{-/-}$(Table 1). This frequency falls below the expected Mendelian ratio of inheritance for the mutant Ron allele $\left(P<0.003\right.$ in $\chi^{2}$ analysis). The observation that the ratio of $\mathrm{Ron}^{+/-}$to $\mathrm{Ron}^{+/+}$is approximately 2:1 suggests that there is little or no loss of $\mathrm{Ron}^{+/-}$embryos at this developmental stage. This was further determined by detailed histologic analyses of the uterine contents of pregnant mice from hemizygous matings. At E6.5, 88 implantation sites were analyzed by complete serial section analysis of the harvested uterine specimens. These analyses showed that despite the evidence of decidualization, only 82 sites were found to contain embryos. The other sites showed no residual evidence of any embryonic structures, suggesting that despite induction of the decidual reaction in the uterine mucosa, the embryos failed to successfully implant. Of the identified embryos, none showed any degenerative changes or histologic abnormalities. The presence of Ron $/-$ embryos at E5.5 and E6.5 demonstrates that Ron $^{-/}$embryos are able to implant and initiate decidualization of the uterus, but loss of the null embryos appears to start to occur shortly after the initiation of implantation.

Prenatal death is observed in various genetic backgrounds. To explore the potential influence of genetic background on the $\mathrm{Ron}^{-/-}$phenotype, chimeric founders were crossed with CD-1 females, generating $\mathrm{Ron}^{+/}$offspring with a mixed genetic background of CD-1/129. When these heterozygous siblings were mated, it was again found that $\mathrm{Ron}^{+/+}$and $\mathrm{Ron}^{+/-}$offspring were present at a ratio of approximately $1: 2$, but no term $\mathrm{Ron}^{-/-}$mice were present (Table 1). This intrauterine death was also observed in mice with a highly enriched C57/Bl6 background ( 6 generations of backcrossing). Therefore, the intrauterine death of $\mathrm{Ron}^{-/-}$embryos is consistently observed across various genetic backgrounds.

Ron is expressed at the blastocyst stage. The fact that Ron ${ }^{-1-}$ embryos fail around E6.5 would imply that Ron is both present and of biologic importance prior to this point in development. To determine if Ron is expressed prior to this developmental roadblock, Ron expression was examined by in situ hybridization. At E3.5, blastocysts are composed of 2 distinct cell types: the cells of the trophectoderm and those of the inner cell mass. Ron expression was observed in the trophectodermal cells, a cell population that represents the earliest extraembryonic structure (Figure 2, a and b). The inner cell mass, which contributes to the embryo proper, was completely devoid of Ron expression. This exclusively extraembryonic trend of Ron expression continued at least through E7.5 of peri-implantation development (Figure 2). Therefore, Ron expression commences as early as E3.5, which precedes the developmental failure of Ron ${ }^{-/-}$embryos.

HGFL is present in the uterus at E4.5-E6.5. Based on the fact that $\mathrm{Ron}^{-/-}$embryos fail prior to E6.5, it was of interest to determine if the ligand for Ron, HGFL, is present in uterine tissue at this stage. Expression of HGFL was measured by Western blot analysis of uterine tissue from pregnant wild-type females at 4.5 and 6.5 days postcoitus, and from virgin females (Figure 3a). HGFL is present in mouse uterine tissue at both days 4.5 and 6.5 of pregnancy. The nongravid uterine tissue was devoid of detectable HGFL. Although these results do not establish that HGFL is synthesized within uterine tissue, it is clear that HGFL is present in the uterine environment at a time when Ron is expressed within the embryonic trophectoderm and when development is terminated in Ron $^{-/-}$embryos. Therefore, a critical developmental signaling system may link maternal HGFL and embryonic Ron. 
a

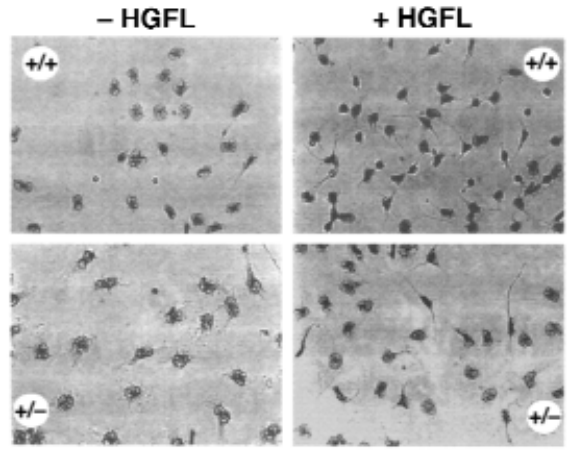

c

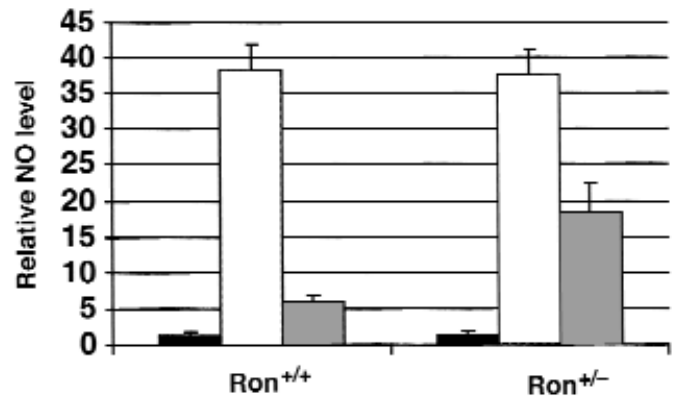

b

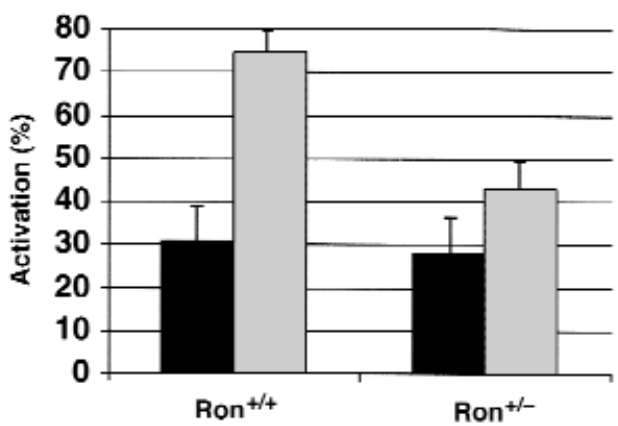

d

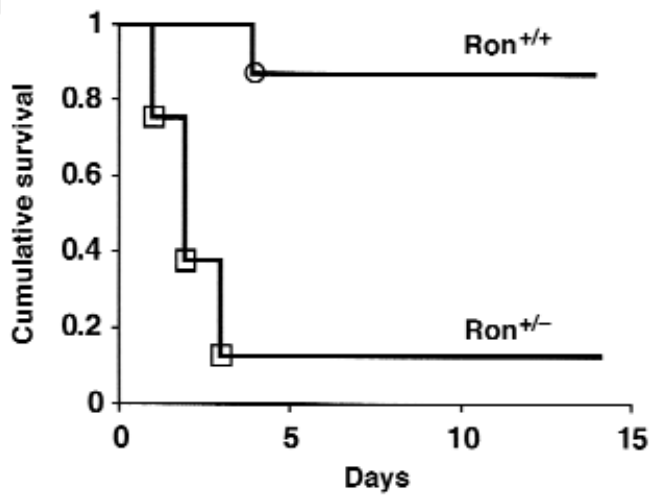

Figure 4

Analysis of macrophage activation, NO synthesis, and septic shock. (a) Resident peritoneal macrophages isolated from Ron ${ }^{+/+}$or Ron $/$- mice (-HGFL) were treated with $100 \mathrm{ng} / \mathrm{mL}$ recombinant human HGFL (+HGFL) and analyzed for morphologic changes, including nuclear condensation, spindle formation, and cytoplasmic elongations. (b) Resident peritoneal macrophages were stimulated with media containing $100 \mathrm{ng} / \mathrm{mL}$ recombinant human HGFL (gray bars), or with control media (black bars). Macrophage activation was determined by blinded counting at 1-3 hours following HGFL treatment. Results are presented as the number of activated macrophages per total number of macrophages. Error bars represent the SD $(P<$ 0.009 , calculated using the Mann-Whitney test; $n=12$ for each genotype; each experiment was repeated 3 times). (c) Resident peritoneal macrophages were stimulated with $1 \mu \mathrm{g} / \mathrm{mL}$ LPS and $100 \mathrm{U} / \mathrm{mL} \mathrm{IFN}-\gamma$ in the absence (hatched bars) or presence (gray bars) of $100 \mathrm{ng} / \mathrm{mL}$ recombinant human HGFL, or left untreated (black bars). All NO values were calculated in reference to the amount of NO in untreated wild-type macrophages, which was set at a value of 1 . The levels of $\mathrm{NO}$ in untreated macrophages from $\operatorname{Ron}^{+/+}$and $\operatorname{Ron}^{+/-}$mice did not differ significantly. All values represent the average values obtained from 3 sets of mice ( $n=12$ for each genotype). Each experiment was assayed in triplicate. Error bars represent the SD $(P<$ 0.03, calculated using the Mann-Whitney test). (d) Increased susceptibility of Ron ${ }^{+/-}$mice to LPS-induced septic shock. Ron ${ }^{+/+}$or Ron ${ }^{+/-}$mice were administered $25 \mu \mathrm{g} / \mathrm{g}$ LPS by peritoneal injection and then monitored daily for mortality over a 14-day period. Results are presented as "cumulative survival," which is the number of surviving mice per total number of mice treated with LPS ( $n=8$ for each genotype). The 2 groups of mice were compared by Kaplan-Meier survival analysis and were found to be statistically different, with a $P$ value of 0.0011 by the Mantel-Cox test.

Defects in morphologic changes in macrophages in Ron ${ }^{+-}$mice. $\mathrm{Ron}^{+/-}$mice displayed no obvious abnormalities, as determined by gross morphology, weight gain, survival, and fertility (data not shown). Ron was expressed at approximately $75 \%$ of wild-type levels in $\mathrm{Ron}^{+/}$- mouse liver (Figure 3b). Similar levels were seen in colon and lung tissue from $\mathrm{Ron}^{+/-}$ mice (data not shown). Therefore, it appears that a reduction in Ron gene dosage does not present a significant liability to the overall health of unchallenged mice. To explore the possibility of a gene dosage effect on specific cellular functions, macrophage activation was compared in $\mathrm{Ron}^{+/+}$ and $\mathrm{Ron}^{+/-}$mice. The total number of resident peritoneal macrophages derived from $\mathrm{Ron}^{+/-}$mice did not differ from the number derived from $\mathrm{Ron}^{+/+}$mice (data not shown). However, macrophages derived from $\mathrm{Ron}^{+/+}$and $\mathrm{Ron}^{+/-}$mice differed dramatically in regard to HGFL-produced macrophage activation, as judged by morphologic changes during activation from cells with large, round nuclei to spindle-shaped cells with condensed, dark nuclei (Figure 4a). Without stimulation by HGFL, the background level of macrophage activation was similar in macrophages derived from $\mathrm{Ron}^{+/+}$and $\mathrm{Ron}^{+/-}$mice (Figure 4, a and b). The presence of HGFL stimulated an increase in the number of activated macrophages in both $\mathrm{Ron}^{+/+}$and $\mathrm{Ron}^{+/-}$cultures. However, the level of HGFL-induced macrophage activation was dependent on the dosage of the Ron gene, with considerably fewer $\mathrm{Ron}^{+/-}$macrophages becoming activated in response to HGFL. The differential in the number of activated macrophages was maintained through a 24-hour period, suggesting that the difference in levels of activation was not due to a delay in the response of $\mathrm{Ron}^{+/-}$ macrophages to HGFL. In addition, HGFL-activated $\mathrm{Ron}^{+/-}$ macrophages are morphologically distinct from their wildtype counterparts in that they do not condense or form spindles to the same degree as the wild-type macrophages; however, they still extend long cytoplasmic processes similar to those of the wild-type macrophages (Figure 4a).

Defective NO regulation and increased susceptibility to septic shock in Ron ${ }^{+/}$mice. To determine whether Ron gene dosage altered HGFL-induced NO synthesis, the levels 
of nitrite (a stable by-product of $\mathrm{NO}$ ) released into the media were compared in cultures of peritoneal macrophages derived from $\mathrm{Ron}^{+/+}$or $\mathrm{Ron}^{+/-}$mice. LPS in the presence of INF- $\gamma$ increased the levels of NO released by macrophages almost 40 -fold, regardless of whether the cells were derived from $\mathrm{Ron}^{+/+}$or $\mathrm{Ron}^{+/-}$mice (Figure $4 \mathrm{c})$. The presence of HGFL reduced NO release markedly. However, the HGFL-mediated suppression of NO release was distinctly dependent on gene dosage, with $\mathrm{Ron}^{+/+}$macrophages releasing far less NO than $\mathrm{Ron}^{+/-}$ macrophages in the presence of HGFL.

NO plays a critical role in the inflammatory response, and elevated NO levels have been implicated in tissue damage and endotoxic shock in mice. Therefore, $\mathrm{Ron}^{+/-}$ mice were tested for their susceptibility to LPS-induced death. Treatment with LPS was not lethal to the majority of $\mathrm{Ron}^{+/+}$mice, with $87.5 \%$ of $\mathrm{Ron}^{+/+}$mice surviving beyond 14 days (Figure 4d). In striking contrast, this same dose of LPS was lethal to the majority of $\mathrm{Ron}^{+/-}$ mice, with only $12.5 \%$ of Ron $^{+/-}$mice surviving.

HGFL expression is unaltered in Ron ${ }^{+/-}$mice. To determine if the there is any compensatory upregulation of HGFL expression in $\mathrm{Ron}^{+/-}$mice, hepatic expression of HGFL was examined in both $\mathrm{Ron}^{+/+}$and $\mathrm{Ron}^{+/-}$mice. Northern blot analysis of hepatic RNA demonstrated that expression of HGFL is essentially identical in $\mathrm{Ron}^{+/+}$and $\mathrm{Ron}^{+/-}$ mice (Figure 3c). Therefore, differences observed in macrophage function are not related to altered levels of HGFL in $\mathrm{Ron}^{+/-}$mice.

\section{Discussion}

Disruption of the gene encoding Ron causes early embryonic death in mice, an outcome quite different than that resulting from the targeted loss of its only known ligand, HGFL (24). HGFL deficiency is compatible with normal development, growth, and reproduction, whereas the loss of its receptor, Ron, results in termination of embryonic development around the time of embryo implantation in the uterine wall. There are at least three possible explanations for some seemingly paradoxical findings. First, another ligand may exist that binds to and activates Ron. HGF does not appear to bind to Ron in vitro (28), so it is unlikely that HGF is compensating for the loss of HGFL. However, other members of the HGF/HGFL family of growth factors are likely to exist, based on the fact that Xenopus has been shown to carry a third distinct HGF/HGFL family member known as livertine (29). Therefore, it is conceivable that a murine homologue of livertine activates Ron and is sufficient to sustain development in HGFL-deficient mice. Second, a low level of constitutive Ron activation is able to sustain an $\mathrm{HGFL}^{-/-}$embryo through critical developmental milestones, a biochemical process that does not occur in $\mathrm{Ron}^{-/-}$embryos. Third, Ron may heterodimerize with multiple receptor partners, and in the early embryo this heterodimerization may be required to respond to other signals; disruption of Ron-dependent, HGFL-independent signal transduction pathways could affect early development processes in Ron $^{-/-}$embryos. However, the association of Ron with other cell surface receptors has not been reported or thoroughly investigated. Note that all of these proposed hypotheses are consistent with the notion that the absence of the receptor is a more stringent biologic hurdle than the absence of the ligand. It has been observed that overexpression of Ron or the expression of constitutively active forms of Ron in tumor cell lines can increase the invasiveness of the cells, implying that Ron may play a general role in invasive cell migration (12-13). The trophectoderm is a highly invasive tissue, initially invading the uterine epithelium during implantation and then sending its invasive fingerlike projections into the uterine stroma. Ron is expressed exclusively in the trophectoderm of the mouse blastocyst (Figure 2), and its presence appears to be required for some critical function of this extraembryonic tissue in the peri-implantation period of development. It may be that Ron is required at this early stage of development to mediate the invasiveness, motility, or growth of embryonic trophectodermal cells, and is essential for maturation within the uterine wall. However, based on the presence of Ron $^{-/-}$embryos at E5.5, it is clear that Ron is not strictly required for the initial phase of implantation.

The onset of Ron expression occurs as early as the blastocyst stage of development in mice, and studies performed in Xenopus and chick embryos have also reported expression of Ron in early embryos, with prominent expression in axial structures of primitive streak-stage embryos (30-32). It was suggested, therefore, that Ron contributes to gastrulation or to the further development of the presumptive neural plate in chick and Xenopus embryos. Mouse embryo gastrulation commences at E5.5, near the gestational period where $\mathrm{Ron}^{-/}$embryos appear to fail. However, mouse Ron expression was not detected in the cells of the embryo proper in this time frame. Rather, Ron expression was strictly localized to the extraembryonic tissues, suggesting that at least in the mouse, Ron may contribute exclusively to development within the extraembryonic tissues or mediate the establishment of a functional interface between maternal and embryonic tissues.

In contrast to the results presented here, indicating that Ron is essential for early embryonic development, it was reported by Correll et al. that homozygous Ron gene disruption in mice was compatible with development to term, growth to adulthood, and reproduction (27). However, the homozygous Ron knockout mice used in that study were more susceptible than wild-type mice to endotoxic shock, similar to the response reported here in the hemizygous Ron ${ }^{+/}$mice. Although the biologic basis for the different phenotypic findings in our studies and the studies of Correll et al. is not yet clear, a number of hypotheses can be proposed. First, the underlying genetic background may dramatically alter the phenotype of mutant mice. In this case, genetic background cannot account for the phenotypic differences in Ron-deficient mice in our study and those of Correll et al. When analyzed in both a Black Swiss/129 hybrid background and a CD-1/129 hybrid background (that used by Correll et al.), we find that Ron $/-$ embryos uniformly fail to develop to term. An alternative explanation is that the phenotypic differences observed are due to the different targeting strategies employed. The targeting vector reported here replaces exons 1-15 (of 19 exons; ref. 15), eliminating the coding sequences for the entire extracellular domain, 
transmembrane domain, and a portion of the intracellular domain. Furthermore, it eliminates the possibility of expression of any of the Ron splice variants that have been described, including one that begins in exon 10 (15), which produces a truncated Ron protein that contains constitutive tyrosine kinase activity (12). In contrast to our targeting strategy, the targeting vector created by Correll et al. was designed to replace approximately 850 bp of exon 1 with a $\beta$-galactosidase expression cassette, leaving the promoter region intact, as well as the gene sequences encoding a large portion of the ligand-binding domain, the transmembrane domain, and the intracellular tyrosine kinase domain. It is therefore possible that one or more splice variants may have been expressed from the mutated Ron gene with residual biologic activity that is sufficient to support embryonic development and growth. Nevertheless, the level or activity of the putative mutant Ron gene product may be insufficient to protect the animals from acute challenges, such as LPS-induced shock. Another possibility is that the removal of exons $1-15$, as well as a small amount of the $5^{\prime}$ flanking sequence of the Ron gene, affects the regulation of a neighboring gene. Although this cannot be ruled out by the studies reported here, the fact that Ron is expressed during mouse development at the time when death occurs is consistent with this gene being responsible for the defect. Additionally, in vitro studies indicate that Ron and its ligand are responsible for the mobility and invasiveness of specific cell types. This is again consistent with a potential defect in implantation, where the invasiveness of trophoblasts is essential.

Because one copy of the Ron allele is maintained and expressed in $\mathrm{Ron}^{+/-}$mice, a dose-dependent effect of the loss of Ron is demonstrated. This underscores the importance of Ron expression in the finely tuned regulation of macrophage-mediated inflammatory responses. Results presented herein demonstrate that Ron may limit the extent of inflammation by limiting the production of $\mathrm{NO}$, one of the principle mediators of the inflammatory, microbicidal, and tumoricidal activity of macrophages, thus protecting tissues from the potentially damaging effects of $\mathrm{NO}$ and inflammation. Consistent with the hypothesis that Ron is a regulator of the macrophage-mediated inflammatory response is the fact that expression of the Ron ligand, HGFL, increases upon injury to the lungs and liver $(33,34)$.

$\mathrm{Ron}^{+/-}$mice are highly sensitive to death by septic shock, a systemic inflammatory response to microbial toxins such as LPS. During this process, there is a catastrophic loss of blood pressure, and oxygen-deprived organs fail. Because NO decreases vascular tone, suppresses cardiac myocytes, and is produced in high amounts by the enzyme iNOS in response to systemic infection, it is thought to be a central factor in the progression of an inflammatory response to septic shock. It is proposed that one function of Ron may be to temper NO production, perhaps by impairing the microbicidal effects of macrophage-mediated inflammation. Because $\mathrm{Ron}^{+/}$ mice display no other obvious abnormality, the gene dose-dependent effects of Ron loss may only be appreciable under conditions of acute challenge or stress.

Because of the early death of Ron ${ }^{-/}$embryos, the role of
Ron in later developmental processes such as bone remodeling, hematopoiesis, megakaryocyte maturation, gastrointestinal and skin development, as well as its function in the developing nervous system, must be illuminated using alternative strategies. Conditional Ron knockouts, whereby Ron gene expression is genetically denied at a specific developmental stage and/or within a specific tissue, would be useful in defining the role of this tyrosine kinase receptor in later developmental events, wound repair, inflammation, and disease processes.

\section{Acknowledgments}

The authors would like to acknowledge Karen Yager, Nancy Roszell, Michael Hughes, Laura Eaton, Melissa Burkart, Lisa Artmayer, Pam Groen, Kathy Saalfeld, and Alicia Emley for technical assistance; Gary Keller for excellence in animal care and maintenance; and Kenn Holmback, Jorge Bezerra, Tom Doetchman, and Thomas Bugge for insightful suggestions and discussion. This work was supported in part by grant DK47003 from the National Institutes of Health (NIH), (to S.J.F. Degen), training grant HL-07527 from the NIH (to R.S. Muraoka), National Research Service Award Postdoctoral Fellowship DK-09466 (to S.E. Waltz), and a Board of Trustees grant from the Children's Hospital Research Foundation (to S.E. Waltz).

1. Bottaro, D.P., et al. 1991. Identification of the hepatocyte growth factor receptor as the c-met proto-oncogene. Science. 251:802-804.

2. Naldini, L., et al. 1991. Scatter factor and hepatocyte growth factor are indistinguishable ligands for the Met receptor. EMBO J. 10:2867-2878.

3. Ronsin, C., Muscatelli, F., Mattei, M.G., and Breathnach, R. 1993. A novel putative receptor tyrosine kinase of the Met family. Oncogene. 8:1195-1202.

4. Gaudino, G., et al. 1994. Ron is a heterodimeric tyrosine kinase receptor activated by the HGF homologue MSP. EMBO J. 13:3524-3532.

5. Schmidt, C., et al. 1995. Scatter factor/hepatocyte growth factor is essential for liver development. Nature. 373:768-771.

6. Uehara, Y., et al. 1995. Placental defect and embryonic lethality in mice lacking HGF/SF. Nature. 373:702-705.

7. Bladt, F., Riethmacher, D., Isenmann, S., Aguzzi, A., and Birchmeier, C. 1995. Essential role for the c-met receptor in migration of myogenic precursor cells into the limb bud. Nature. 376:768-771.

8. Degen, S.J.F., Stuart, L.A., and Jamison, C.S. 1991. Characterization of the mouse cDNA and gene coding for a hepatocyte growth factor-like protein: expression during development. Biochemistry. 30:9781-9791.

9. Han, S., Stuart, L.A., and Degen, S.J.F. 1991. Characterization of the DNF15S2 locus on human chromosome 3: identification of a gene coding for four kringle domains with homology to hepatocyte growth factor. Biochemistry. 30:9768-9780.

10. Bezerra, J.A., Han, S., Danton, M.J.S., and Degen, S.J.F. 1993. Are hepatocyte growth factor-like protein and macrophage stimulating protein the same protein? Protein Sci. 2:666-668.

11. Yoshimura, T., Yuhki, N., Wang, M., Skeel, A., and Leonard, E.J. 1993. Cloning, sequencing and expression of human macrophage stimulating protein (MSP, MST1) confirms MSP as a member of the family of kringle proteins and locates the MSP gene on chromosome 3. J. Biol. Chem. 268:15461-15468

12. Collesi, C., Santoro, M.M., Gaudino, G., and Comoglio, P.M. 1996. A splicing variant of the Ron transcript induces constitutive tyrosine kinase activity and an invasive phenotype. Mol. Cell. Biol. 16:5518-5526.

13. Santoro, M.M., Collesi, C., Grisendi, S., Gaudino, G., and Comoglio, P.M. 1996. Constitutive activation of the Ron gene promotes invasive growth but not transformation. Mol. Cell. Biol. 16:7072-7083.

14. Wang, M.-H., Skeel, A., and Leonard, E.J. 1996. Proteolytic cleavage and activation of pro-macrophage-stimulating protein by resident peritoneal macrophage. J. Clin. Invest. 97:720-727.

15. Waltz, S.E., et al. 1998. Characterization of the mouse Ron tyrosine kinase gene. Oncogene. 16:27-42.

16. Iwama, A., Okano, K., Sudo, T., Matsuda, Y., and Suda, T. 1994. Molecular cloning of a novel receptor tyrosine kinase gene, STK, derived from enriched hematopoietic stem cells. Blood. 83:3160-3169.

17. Iwama, A., et al. 1995. Terminal differentiation of murine resident peritoneal macrophages is characterized by expression of the STK receptor tyrosine kinase, a receptor for macrophage stimulating protein. Blood. 86:3394-3403.

18. Banu, N., et al. 1996. Modulation of megakaryocytopoiesis by human macrophage stimulating protein, the ligand for the Ron receptor. $J$. 
Immunol. 156:2933-2940.

19. Kurihara, N., Iwama, A., Tatsumi, J., Ikeda, K., and Suda, T. 1996. Macrophage-stimulating protein activates STK receptor tyrosine kinase on osteoclasts and facilitates bone resorption by osteoclast-like cells. Blood. 87:3704-3710.

20. Leonard, E.J., and Skeel, A. 1976. A serum protein that stimulates macrophage movement, chemotaxis, and spreading. Exp. Cell Res. 102:434-438.

21. Wang, M.-H., et al. 1994. Macrophage-stimulating protein inhibits induction of nitric oxide production by endotoxin- or cytokine-stimulated mouse macrophages. J. Biol. Chem. 269:14027-14031.

22. Gaudino, G., et al. 1995. The proto-oncogene Ron is involved in development of epithelial, bone, and neuroendocrine tissues. Oncogene. 11:2627-2637.

23. Quantin, B., Schuhbaur, B., Gesnel, M.C., Dolle, P., and Breathnach, R. 1995. Restricted expression of the ron gene encoding the macrophage stimulating protein receptor during mouse development. Dev. Dyn. 20:383-390.

24. Bezerra, J.A., Carrick, T.L., Degen, J.L., Witte, D.P., and Degen, S.J.F. 1998 Biological effects of the targeted loss of hepatocyte growth factor-like protein in mice. J. Clin. Invest. 101:1175-1183.

25. Witte, D.P., et al. 1993. Platelet activation releases megakaryocyte-synthesized apolipoprotein-J, a highly abundant protein in atheromatous lesions. Am. J. Pathol. 143:763-773.

26. Waltz, S.E., et al. 1997. Functional characterization of the domains con- tained in hepatocyte growth factor-like protein. J. Biol. Chem. 272:30526-30537.

27. Correll, P.H., et al. 1997. Deregulated inflammatory response in mice lacking the STK/RON receptor tyrosine kinase. Genes Funct. 1:69-83.

28. Wang, M.-H., et al. 1994. Identification of the Ron gene product as the receptor for the human macrophage-stimulating protein. Science. 266:117-119.

29. Altaba, A.R., and Thery, C. 1996. Involvement of livertine, a hepatocyte growth factor family member, in neural morphogenesis. Mech. Dev. 60:207-220.

30. Aberger, F., Schmidt, G., and Richter, K. 1996. The Xenopus homologue of hepatocyte growth factor-like protein is specifically expressed in the presumptive neural plate during gastrulation. Mech. Dev. 54:23-37.

31. Nakamura, T., et al. 1996. Cloning and expression of Xenopus HGF-like protein (HLP) and Ron/HLP receptor implicate their involvement in early neural development. Biochem. Biophys. Res. Commun. 224:564-573.

32. Thery, C., Sharpe, M.J., Batley, S.J., Stern, C.D., and Gherardi, E. 1995. Expression of HGF/SF, HGFL/MSP and of Met suggests new functions during development. Dev. Genet. 17:90-101.

33. Willett, C.G., et al. 1997. Differential screening of a human chromosome 3 library identifies hepatocyte growth factor-like/macrophage stimulating protein and its receptor in injured lung. J. Clin. Invest. 99:2979-2991.

34. Bezerra, J.A., Laney, D.W., Jr., and Degen, S.J.F. 1994. Increased expression of mRNA for hepatocyte growth factor-like protein during liver regeneration and inflammation. Biochem. Biophys. Res. Commun. 203:666-673. 